\title{
On the generalization of the D. H. Lehmer problem II
}

by

\author{
YAMING LU and YUAN Yi (Xi'an)
}

1. Statement of the main result. Let $q \geq 3$ be a positive odd integer. For an integer $a$ with $1 \leq a \leq q$ and $(a, q)=1$, we write $\bar{a}$ for the integer satisfying $1 \leq \bar{a} \leq q, a \bar{a} \equiv 1(\bmod q)$. The classical Lehmer problem is to study the nontrivial properties of

$$
r(q)=\sum_{\substack{1 \leq a \leq q \\ 2 \nmid a+\bar{a}}}^{\prime} 1,
$$

where the dash means that the sum runs through the integers $a$ which are coprime to $q$. W. P. Zhang ([4], [5]) gave an asymptotic formula for $r(q)$ :

$$
r(q)=\frac{1}{2} \phi(q)+O\left(q^{1 / 2} d^{2}(q) \log ^{2} q\right),
$$

where $\phi(q)$ and $d(q)$ are the Euler function and divisor function, respectively. In 1994, Zhang [6] proved

$$
\begin{aligned}
M(q, k) & :=\sum_{\substack{1 \leq a \leq q \\
2 \mid a+\bar{a}+1}}^{\prime}(a-\bar{a})^{2 k} \\
& =\frac{\phi(q) q^{2 k}}{(2 k+1)(2 k+2)}+O\left(4^{k} q^{(4 k+1) / 2} d^{2}(q) \log ^{2} q\right),
\end{aligned}
$$

where $k$ is a nonnegative integer.

The formula (1) was recently generalized by the present authors. Let $n \geq 2$ be a fixed positive integer and let $q \geq 3$ and $c$ be two integers with $(n, q)=(c, q)=1$. Denote

$$
r_{n}\left(\delta_{1}, \delta_{2}, c ; q\right)=\sum_{\substack{a \leq \delta_{1} q \\ a b \equiv c(\bmod q) \\ n \nmid a+b}}^{\prime} \sum_{\substack{b \leq \delta_{2} q \\ n \nmid a+b}}^{\prime} \quad\left(0<\delta_{1}, \delta_{2} \leq 1\right) .
$$

2010 Mathematics Subject Classification: Primary 11N69.

Key words and phrases: Lehmer problem, mean value, Gauss sums. 
Then

$$
r_{n}\left(\delta_{1}, \delta_{2}, c ; q\right)=\left(1-\frac{1}{n}\right) \delta_{1} \delta_{2} \phi(q)+O\left(q^{1 / 2} d^{6}(q) \log ^{2} q\right) .
$$

In this paper, we deal with the Lehmer problem weighted by $\left|a-\bar{a}_{c}\right|^{\alpha}$ for $\alpha>0$, where $\bar{a}_{c}$ is the unique integer satisfying $1 \leq \bar{a}_{c} \leq q$ and $a \bar{a}_{c} \equiv c$ $(\bmod q)$. We will give an asymptotic formula for it:

TheOREM. Let $n \geq 2$ be a fixed positive integer, $q \geq 3$ and $c$ be two integers with $(n, q)=(c, q)=1$, and $d$ be an integer with $1 \leq d \leq n$. For any integer $M$ and a positive integer $N$, we write

$$
\mathcal{L}=\{a: M+1 \leq a \leq M+N,(a, q)=1\}
$$

and for $\alpha>0$ define

$$
S(\alpha, q):=\sum_{\substack{a, b \in \mathcal{L} \\ a b \equiv c(\bmod q) \\ a+b \equiv d(\bmod n)}}|a-b|^{\alpha} .
$$

Then

$$
S(\alpha, q)=\frac{2 \phi(q)}{(\alpha+1)(\alpha+2) n q^{2}} N^{\alpha+2}+O\left(q^{1 / 2+\varepsilon} N^{\alpha}\left(N q^{-1}+1\right)\right),
$$

where the $O$ constant depends on $n, \alpha$ and $\varepsilon$.

Note. In the special case of $n=2$ and $d=1$ (which amounts to the problem in (2)), we can find that $a \neq b$ in the sum in (4), and thus our result also holds for $\alpha=0$.

Notation. We set $e(x)=e^{2 \pi i x}$. For $\chi$ the Dirichlet character modulo $q$, we denote by $G(m, \chi)=\sum_{n \leq q,(n, q)=1} \chi(n) e(m n / q)$ the Gauss sum. We write $\|x\|$ for the distance of $x$ from the nearest integer. $\varepsilon$ always denotes a sufficiently small positive real number which can be different at each occurrence. We will use $d(q) \ll q^{\varepsilon}$ throughout the paper without explicit statement.

2. Auxiliary lemmas. All the lemmas introduced here will be used in estimating the error term of $S(\alpha, q)$.

LEMma 1 ([3, §5.1, Lemma 3]). Assume that $U$ is a positive real number, $K_{0}$ is an integer, $K$ is a positive integer, and that $\alpha$ and $\beta$ are real numbers. If $\alpha$ can be written in the form

$$
\alpha=\frac{s}{r}+\frac{\theta}{r^{2}}, \quad(r, s)=1, r \geq 1,|\theta| \leq 1
$$

we have

$$
\sum_{k=K_{0}+1}^{K_{0}+K} \min \left(U, \frac{1}{\|\alpha k+\beta\|}\right) \ll\left(\frac{K}{r}+1\right)(U+r \log r) .
$$


Lemma 2 ([2, Lemma 4]). Suppose that $q$ and $c$ are integers satisfying $q \geq 3$ and $(c, q)=1$. Then for any integers $k_{1}, k_{2}$, we have

$$
\sum_{\substack{\chi \bmod q \\ \chi \neq \chi^{0}}} \bar{\chi}(c) G\left(k_{1}, \chi\right) G\left(k_{2}, \chi\right) \ll \phi(q) q^{1 / 2} d(q) \min \left\{\left(k_{1}, q\right),\left(k_{2}, q\right)\right\} .
$$

3. Proof of the Theorem. It is obvious that

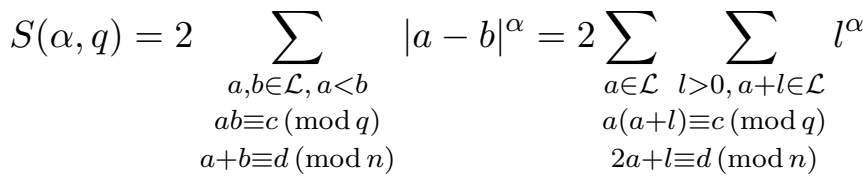

$$
\begin{aligned}
& =\frac{2}{\phi(q)} \sum_{\substack{a \in \mathcal{L} \\
2 a+l \equiv d(\bmod n)}} \sum_{\substack{l>0, a+l \in \mathcal{L} \\
\chi}} \bar{\chi}(c) \chi(a(a+l)) \\
& =\frac{2}{\phi(q)} \sum_{a \in \mathcal{L}} \sum_{l>0, a+l \in \mathcal{L}} l^{\alpha} \\
& 2 a+l \equiv d(\bmod n)
\end{aligned}
$$

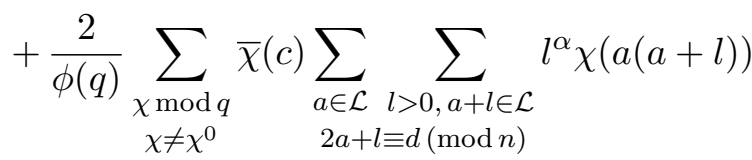

$$
\begin{aligned}
& =S+E
\end{aligned}
$$

say. Here

$$
\begin{aligned}
S & =\frac{2}{\phi(q)} \sum_{\substack{a \in \mathcal{L} \\
2 a+l \equiv d(\bmod n)}} \sum_{\substack{l>0, a+l \in \mathcal{L}\\
}} l^{\alpha} \sum_{\substack{M+1 \leq a \leq M+N \\
(a, q)=1}} l_{\substack{l \leq M+N-a \\
l \equiv d-2 a(\bmod n) \\
(a+l, q)=1}} \sum^{\alpha} \sum_{\substack{(q)\\
}} \mu(m) \sum_{\substack{l \leq M+N-a \\
l \equiv d-2 a(\bmod n) \\
l \equiv-a(\bmod m)}} l^{\alpha} .
\end{aligned}
$$

From $(n, q)=1$ we know that there is a $d^{\prime}$ such that

$$
S=\frac{2}{\phi(q)} \sum_{\substack{M+1 \leq a \leq M+N \\(a, q)=1}} \sum_{m \mid q} \mu(m) \sum_{\substack{l \leq M+N-a \\ l \equiv d^{\prime}(\bmod m n)}} l^{\alpha} .
$$


Partial summation gives

$$
\begin{aligned}
\sum_{\substack{l \leq M+N-a \\
l \equiv d^{\prime}(\bmod m n)}} l^{\alpha} & =\int_{0}^{M+N-a} u^{\alpha} d\left(\sum_{\substack{l \leq u \\
l \equiv d^{\prime}(\bmod m n)}} 1\right) \\
& =(M+N-a)^{\alpha}\left(\frac{M+N-a}{(\alpha+1) m n}+O(1)\right),
\end{aligned}
$$

thus

(9) $S=\frac{2}{\phi(q)} \sum_{M+1 \leq a \leq M+N} \sum_{m \mid q} \mu(m)(M+N-a)^{\alpha}\left(\frac{M+N-a}{(\alpha+1) m n}+O(1)\right)$

$$
\begin{gathered}
=\frac{2}{(\alpha+1) n q} \sum_{\substack{M+1 \leq a \leq M+N \\
(a, q)=1}}(M+N-a)^{\alpha+1} \\
+O\left(\frac{d(q)}{\phi(q)} \sum_{\substack{M+1 \leq a \leq M+N \\
(a, q)=1}}(M+N-a)^{\alpha}\right) .
\end{gathered}
$$

Analogously to (8), we may get

$$
\begin{aligned}
\sum_{\substack{M+1 \leq a \leq M+N \\
(a, q)=1}}(M+N-a)^{\alpha} & =\sum_{m \mid q} \mu(m) \sum_{\substack{M+1 \leq a \leq M+N \\
m \mid a}}(M+N-a)^{\alpha} \\
& =\frac{\phi(q)}{(\alpha+1) q} N^{\alpha+1}+O\left(N^{\alpha} d(q)\right) .
\end{aligned}
$$

Combining with (9), this implies that

$$
S=\frac{2 \phi(q)}{(\alpha+1)(\alpha+2) n q^{2}} N^{\alpha+2}+O\left(N^{\alpha+1} q^{-1+\varepsilon}\right) .
$$

The remaining task is to estimate $E$ :

$$
\begin{aligned}
E= & \frac{2}{\phi(q)} \sum_{\substack{\chi \bmod q \\
\chi \neq \chi^{0}}} \bar{\chi}(c) \sum_{\substack{M+1 \leq a \leq M+N \\
2 a+l \equiv d(\bmod n)}} \sum_{\substack{l \leq M+N-a \\
\phi(q)}} l_{\substack{\chi \bmod q \\
\chi \neq \chi^{0}}} \bar{\chi}(c) \sum_{\substack{M+1 \leq a \leq M+N \\
l \leq M+N-a}} l^{\alpha} \chi(a(a+l)) \\
& \times \sum_{j=1}^{n} e\left(\left(\frac{2 a+l-d}{n}\right) j\right) .
\end{aligned}
$$


Making use of the identity

$$
\chi(a)=\frac{1}{q} \sum_{k=1}^{q-1} G(k, \chi) e\left(-\frac{a k}{q}\right) \quad\left(\chi \neq \chi^{0}\right),
$$

we obtain

$$
\begin{aligned}
& E=\frac{2}{\phi(q) q^{2}} \sum_{\substack{\chi \bmod q \\
\chi \neq \chi^{0}}} \bar{\chi}(c) \sum_{M+1 \leq a \leq M+N} \sum_{\substack{l \leq M+N-a \\
l^{\alpha}}} \sum_{j=1}^{n} e\left(\left(\frac{2 a+l-d}{n}\right) j\right) \\
& \times \sum_{k_{1} \leq q-1} G\left(k_{1}, \chi\right) e\left(-\frac{a k_{1}}{q}\right) \sum_{k_{2} \leq q-1} G\left(k_{2}, \chi\right) e\left(-\frac{a+l}{q} k_{2}\right) \\
& =\frac{2}{\phi(q) q^{2}} \sum_{j \leq n} e\left(-\frac{d}{n} j\right) \sum_{k_{1} \leq q-1} \sum_{k_{2} \leq q-1} \sum_{l \leq N-1} l^{\alpha} e\left(\left(\frac{j}{n}-\frac{k_{2}}{q}\right) l\right) \\
& \times \sum_{M+1 \leq a \leq M+N-l} e\left(\left(\frac{2 j}{n}-\frac{k_{1}+k_{2}}{q}\right) a\right) \sum_{\substack{\chi \bmod q \\
\chi \neq \chi^{0}}} \bar{\chi}(c) G\left(k_{1}, \chi\right) G\left(k_{2}, \chi\right) .
\end{aligned}
$$

Lemma 2 yields

$$
\begin{aligned}
E & \ll q^{-3 / 2} d(q) \sum_{j \leq n} \sum_{k_{1} \leq q-1} \sum_{k_{2} \leq q-1} \min \left\{\left(k_{1}, q\right),\left(k_{2}, q\right)\right\} \\
& \times\left|\sum_{l \leq N-1} l^{\alpha} e\left(\left(\frac{j}{n}-\frac{k_{2}}{q}\right) l\right) \sum_{M+1 \leq a \leq M+N-l} e\left(\left(\frac{2 j}{n}-\frac{k_{1}+k_{2}}{q}\right) a\right)\right| \\
= & q^{-3 / 2} d(q) \sum_{j \leq n}\left(\sum_{\substack{k_{1} \leq q-1 \\
k_{1}+k_{2}=q}} \sum_{\substack{k_{2} \leq q-1 \\
k_{1} \leq q-1 \\
k_{1}+k_{2} \neq q}}+\sum_{k_{2} \leq q-1}\right)=E_{1}+E_{2}
\end{aligned}
$$

say.

We estimate $E_{1}$ first:

(12) $\quad E_{1} \ll$

$$
\begin{aligned}
& q^{-3 / 2} d(q) \sum_{j \leq n} \sum_{k_{1} \leq q-1}\left(k_{1}, q\right)\left|\sum_{l \leq N-1} l^{\alpha} e\left(\left(\frac{j}{n}+\frac{k_{1}}{q}\right) l\right) \sum_{M+1 \leq a \leq M+N-l} e\left(\frac{2 j}{n} a\right)\right| \\
& =q^{-3 / 2} d(q) \sum_{j \leq n} \sum_{k_{1} \leq q-1}\left(k_{1}, q\right)\left|\sum_{l \leq N-1}(N-l) l^{\alpha} e\left(\left(\frac{j}{n}+\frac{k_{1}}{q}\right) l\right)\right| \\
& n \mid 2 j \\
& +q^{-3 / 2} d(q) \sum_{\substack{j \leq n \\
n \nmid 2 j}} \sum_{k_{1} \leq q-1}\left(k_{1}, q\right)\left|\sum_{l \leq N-1} l^{\alpha} e\left(\left(\frac{j}{n}+\frac{k_{1}}{q}\right) l\right) \frac{e(2 j(N-l) / n)-1}{1-e(2 j / n)}\right| .
\end{aligned}
$$


Notice that $k_{1} / q \pm l / n \neq 0$ for $j \leq n$ and $k_{1} \leq q-1$ since $(n, q)=1$, we obtain by partial summation

$$
\sum_{l \leq N-1}(N-l) l^{\alpha} e\left(\left(\frac{j}{n}+\frac{k_{1}}{q}\right) l\right) \ll N^{\alpha+1} \frac{1}{\left\|\frac{k_{1}}{q}+\frac{j}{n}\right\|},
$$

and also

$$
\sum_{l \leq N-1} l^{\alpha} e\left(\left(\frac{j}{n}+\frac{k_{1}}{q}\right) l\right)\left(e\left(\frac{2 j(N-l)}{n}\right)-1\right) \ll N^{\alpha}\left(\frac{1}{\left\|\frac{k_{1}}{q}+\frac{j}{n}\right\|}+\frac{1}{\left\|\frac{k_{1}}{q}-\frac{j}{n}\right\|}\right) .
$$

Combining these with (12) implies

$$
\begin{aligned}
E_{1} \ll q^{-3 / 2} d(q) N^{\alpha+1} \sum_{\substack{j \leq n \\
n \mid 2 j}} \sum_{k_{1} \leq q-1}\left(k_{1}, q\right) \frac{1}{\left\|\frac{k_{1}}{q}+\frac{j}{n}\right\|} \\
+q^{-3 / 2} d(q) N^{\alpha} \sum_{\substack{j \leq n \\
n \nmid 2 j}} \frac{1}{\|2 j / n\|} \sum_{k_{1} \leq q-1}\left(k_{1}, q\right)\left(\frac{1}{\left\|\frac{k_{1}}{q}+\frac{j}{n}\right\|}+\frac{1}{\left\|\frac{k_{1}}{q}-\frac{j}{n}\right\|}\right) .
\end{aligned}
$$

Here

$$
\begin{aligned}
\sum_{k_{1} \leq q-1} \frac{\left(k_{1}, q\right)}{\left\|\frac{k_{1}}{q} \pm \frac{j}{n}\right\|} & =\sum_{\substack{m \mid q \\
m<q}} \sum_{\substack{k_{1} \leq q-1 \\
\left(k_{1}, q\right)=m}} \frac{\left(k_{1}, q\right)}{\left\|\frac{k_{1}}{q} \pm \frac{j}{n}\right\|} \\
& =\sum_{\substack{m \mid q \\
m<q}} m \sum_{\substack{k \leq(q-1) / m \\
(k, q)=1}} \frac{1}{\left\|\frac{m k}{q} \pm \frac{j}{n}\right\|} \\
& =\sum_{\substack{m \mid q \\
m<q}} m \sum_{h \mid q} \mu(h) \sum_{k \leq(q-1) /(m h)} \frac{1}{\left\|\frac{m h k}{q} \pm \frac{j}{n}\right\|} .
\end{aligned}
$$

Since $\|m h k / q \pm j / n\| \gg m / q$ with the $\gg$ constant depending on $n$, we have

$$
\sum_{k_{1} \leq q-1} \frac{\left(k_{1}, q\right)}{\left\|\frac{k_{1}}{q} \pm \frac{j}{n}\right\|} \ll \sum_{\substack{m \mid q \\ m<q}} m \sum_{h \mid q} \sum_{k \leq(q-1) /(m h)} \min \left(\frac{q}{m}, \frac{1}{\left\|\frac{m h k}{q} \pm \frac{j}{n}\right\|}\right) .
$$

We write $m h k / q=k^{\prime} / q^{\prime}$ with $k^{\prime} / q^{\prime}$ a reduced fraction and $q^{\prime} \geq 1$. Then obviously, $q /(m h) \leq q^{\prime} \leq q / m$. With Lemma 1 and (14), this gives

$$
\begin{aligned}
\sum_{k_{1} \leq q-1} \frac{\left(k_{1}, q\right)}{\left\|\frac{k_{1}}{q} \pm \frac{j}{n}\right\|} & \ll \sum_{\substack{m \mid q \\
m<q}} m \sum_{h \mid q}\left(\frac{(q-1) /(m h)}{q^{\prime}}+1\right)\left(\frac{q}{m}+q^{\prime} \log q^{\prime}\right) \\
& \ll q^{1+\varepsilon} .
\end{aligned}
$$


Combining this with (13), we have

$$
E_{1} \ll q^{-1 / 2+\varepsilon} N^{\alpha+1} .
$$

Estimating $E_{2}$ proceeds almost in the same way:

$$
\begin{aligned}
& E_{2}=q^{-3 / 2} d(q) \sum_{j \leq n} \sum_{\substack{k_{1} \leq q-1 \\
k_{1}+k_{2} \neq q}} \sum_{\substack{k_{2} \leq q-1 \\
\text { f }}} \min \left\{\left(k_{1}, q\right),\left(k_{2}, q\right)\right\} \\
& \times\left|\sum_{l \leq N-1} l^{\alpha} e\left(\left(\frac{j}{n}-\frac{k_{2}}{q}\right) l\right) \sum_{M+1 \leq a \leq M+N-l} e\left(\left(\frac{2 j}{n}-\frac{k_{1}+k_{2}}{q}\right) a\right)\right| \\
& =q^{-3 / 2} d(q) \sum_{j \leq n} \sum_{\substack{k_{1} \leq q-1 \\
k_{1}+k_{2} \neq q}} \sum_{\substack{k_{2} \leq q-1 \\
\text { f }}} \min \left\{\left(k_{1}, q\right),\left(k_{2}, q\right)\right\} \\
& \times\left|\sum_{l \leq N-1} l^{\alpha} e\left(\left(\frac{j}{n}-\frac{k_{2}}{q}\right) l\right) \frac{e\left(\left(\frac{2 j}{n}-\frac{k_{1}+k_{2}}{q}\right)(N-l)\right)-1}{1-e\left(\frac{2 j}{n}-\frac{k_{1}+k_{2}}{q}\right)}\right| \\
& \ll q^{-3 / 2} d(q) \sum_{j \leq n} \sum_{\substack{k_{1} \leq q-1 \\
k_{1}+k_{2} \neq q}} \sum_{\substack{k_{2} \leq q-1 \\
\text { f }}} \frac{\min \left\{\left(k_{1}, q\right),\left(k_{2}, q\right)\right\}}{\left\|\frac{k_{1}+k_{2}}{q}-\frac{2 j}{n}\right\|} \\
& \times\left\{\left|\sum_{l \leq N-1} l^{\alpha} e\left(\left(\frac{k_{1}}{q}-\frac{j}{n}\right) l\right)\right|+\left|\sum_{l \leq N-1} l^{\alpha} e\left(\left(\frac{j}{n}-\frac{k_{2}}{q}\right) l\right)\right|\right\} \\
& \ll q^{-3 / 2} d(q) N^{\alpha} \sum_{j \leq n} \sum_{\substack{k_{1} \leq q-1 \\
k_{1}+k_{2} \neq q}} \sum_{\substack{k_{2} \leq q-1 \\
\text { 促 }}} \frac{\min \left\{\left(k_{1}, q\right),\left(k_{2}, q\right)\right\}}{\left\|\frac{k_{1}+k_{2}}{q}-\frac{2 j}{n}\right\|} \\
& \times\left(\frac{1}{\left\|\frac{k_{1}}{q}-\frac{j}{n}\right\|}+\frac{1}{\left\|\frac{k_{2}}{q}-\frac{j}{n}\right\|}\right) \\
& \ll q^{-3 / 2} d(q) N^{\alpha} \sum_{j \leq n} \sum_{k_{1} \leq q-1} \frac{\left(k_{1}, q\right)}{\left\|\frac{k_{1}}{q}-\frac{j}{n}\right\|} \sum_{\substack{k_{2} \leq q-1 \\
k_{1}+k_{2} \neq q}} \frac{1}{\left\|\frac{k_{1}+k_{2}}{q}-\frac{2 j}{n}\right\|} .
\end{aligned}
$$

Making use of (15), we have

$$
E_{2} \ll q^{1 / 2+\varepsilon} N^{\alpha}
$$

By (11), (16) and (18), we obtain $E \ll q^{1 / 2+\varepsilon} N^{\alpha}\left(N q^{-1}+1\right)$. With (6) and (10), this establishes the theorem.

Acknowledgments. The authors would like to express their gratitude to Prof. Wenpeng Zhang for his kind suggestions, and also to the referee for his helpful comments.

This work was supported by N.S.F. No. 10601039 of P.R. China. 


\section{References}

[1] Y. M. Lu and Y. Yi, On the generalization of the D. H. Lehmer problem, Acta Math. Sin. (English Ser.) 25 (2009), 1269-1274.

[2] - - - Partitions involving D. H. Lehmer numbers, Monatsh. Math. 159 (2010), $45-58$.

[3] C. D. Pan and C. B. Pan, Goldbach Conjecture, Science Press, Beijing, 1992.

[4] W. P. Zhang, A problem of D. H. Lehmer and its generalization, Compos. Math. 86 (1993), 307-316.

[5] -, A problem of D. H. Lehmer and its generalization (II), ibid. 91 (1994), 47-56.

[6] -, On the difference between a D. H. Lehmer number and its inverse modulo q, Acta Arith. 68 (1994), 255-263.

Yaming Lu, Yuan Yi

School of Science

Xi'an Jiaotong University

Xi'an, Shaanxi, 710049, P.R. China

E-mail: yaminglu@mail.xjtu.edu.cn

yuanyi@mail.xjtu.edu.cn

Received on 9.4.2009

and in revised form on 27.9.2009 